\title{
Giardia and Cryptosporidium and public health: the epidemiological scenario from the Italian perspective
}

\author{
Annunziata Giangaspero • Federica Berrilli • \\ Olga Brandonisio
}

Received: 28 April 2007 / Accepted: 23 May 2007 / Published online: 26 June 2007

(C) Springer-Verlag 2007

\begin{abstract}
Giardia and Cryptosporidium spp. are protozoa that cause human and animal disease worldwide and often exhibit zoonotic transmission. This review gives ample information concerning the epidemiology of these parasites in Italy, i.e. prevalence data in humans, farm and pet animals, shellfish and aquatic environment. Moreover, it reports genotyping results obtained from different isolates, with particular emphasis on the spread of host-specific and zoonotic species/genotypes of various origin, and on molecular data that make the Italian situation different from that of other countries. Finally, possible explanations are given for the infrequent reports of Giardia and Cryptosporidium spp. outbreaks, despite widespread faecal contamination by these parasites.
\end{abstract}

This work was supported by a research grant for Research of Relevant National Interest (PRIN 2005-2007) from the Ministry of the University and Research, Italy.

\footnotetext{
A. Giangaspero

Dipartimento di Scienze delle Produzioni, dell'Ingegneria e della Meccanica e dell'Economia Applicate ai Sistemi Agro-Zootecnici, University of Foggia,

71100 Foggia, Italy

F. Berrilli

Dipartimento di Sanità Pubblica e Biologia Cellulare,

University of Tor Vergata,

00133 Rome, Italy

O. Brandonisio $(\square)$

Dipartimento di Clinica Medica,

Immunologia e Malattie Infettive, University of Bari,

70124 Bari, Italy

e-mail: brandonisio@midim.uniba.it
}

\section{Introduction}

Giardia and Cryptosporidium are well-known food- and waterborne protozoa affecting humans and a wide range of domestic and wild animals. They are significant etiological agents of intestinal disorders, mainly in immunocompromised subjects. Over the last few decades, the scientific community has focussed much attention on Giardia and Cryptosporidium to understand how they spread in humans, animals and the environment and to figure out whether infected animals can serve as reservoirs of infection for humans. This is especially important because of the latest molecular studies which state that mainly Giardia duodenalis Assemblage A and Cryptosporidium parvum have potential zoonotic relevance due to their wider host range (Fayer 2004; Thompson and Monis 2004; Xiao and Ryan 2004). Hence, mapping and understanding the worldwide epidemiology of these protozoa-from prevalence in humans, animals and the environment to molecular genotyping of isolates - is an urgent task for the scientific community.

Italy (long. $6-18^{\circ} \mathrm{E}$; lat. $47-35^{\circ} \mathrm{N}$ ) is a peninsula in Southern Europe covering $301,302 \mathrm{~km}^{2}$ and surrounded on three sides by the Mediterranean Sea. The country has $58,751,711$ inhabitants and the fifth highest population density in Europe. The largest farm animal population is represented by poultry (171 million), followed by pigs (9.3 million), sheep (6.8 million), cattle (6.3 million), goats (1 million), buffaloes $(250,000)$ and horses $(200,000)$. Pet animals include 6.6 million cats and 5.8 million privately owned dogs (ISTAT 2005).

In recent years, Italian researchers have contributed greatly to knowledge about giardiosis and cryptosporidiosis in the country, and most debated topics have been dealt with. Therefore the present review reports the prevalence 
studies carried out in humans, animals and groundwater, including the molecular background when available, to give a homogeneous picture of the epidemiology of Giardia and Cryptosporidium in a national framework. It also presents the diagnostic tools and clinical aspects. Finally, there is a discussion of the potential impact on public health in comparison with other countries, and the main objectives to be pursued in the future are set out.

\section{Human infection}

Human giardiosis and cryptosporidiosis are not notifiable diseases in Italy, and prevalence data are based on specialised studies, which suggest that $G$. duodenalis and Cryptosporidium spp. are common protozoa in humans (Table 1).

The first indexed reports of human giardiosis in Italy date back to the 1960s (Pucci et al. 1967; De Riu et al. 1967), but only later were larger-scale studies carried out, showing that Giardia clinically affects $3.2 \%$ of adult patients (Libanore et al. 1991) and $0.9 \%$ (Caprioli et al. 1996) to $4.7 \%$ of children (Cevenini et al. 1985; Scotti et al. 1996).

Later on, a significant decline in prevalence from 4.66 to $0.94 \%$ and from 10.99 to $2.41 \%$, independent of age, was registered in a 4-year survey (1994-1998) of hospitalised and non-hospitalised subjects (Ballone et al. 2001), and the latter prevalence was recently confirmed by Capelli et al. (2003) and Crotti et al. (2005).

Subjects at risk include residents in psychiatric institutions and also Human Immunodeficient Virus (HIV) patients. In mental patients, G. lamblia (syn. G. duodenalis) was detected in about $2.5 \%$ of the population in two different studies made in Central (Giacometti et al. 1997) and Northern Italy (Gatti et al. 2000). Although Giardia is not considered a common opportunistic pathogen in immunocompromised patients, the prevalence of giardiosis

Table 1 Range of prevalence (in percentages) of G. duodenalis and Cryptosporidium in different host species in Italy

\begin{tabular}{|c|c|c|}
\hline Host & Giardia & Cryptosporidium \\
\hline Humans & $0.9-6.15$ & $0.84-11$ \\
\hline Dogs & $3.6-80$ & 3.3 \\
\hline Cats & $5.6-15.8$ & - \\
\hline Cattle & $66.6^{\mathrm{a}}$ & $58.3^{\mathrm{a}}$ \\
\hline Sheep & 1.5 & - \\
\hline Water & $30^{\mathrm{a}}$ & $24.4^{\mathrm{a}}$ \\
\hline buffaloes & 18.1 & 14.7 \\
\hline Horse & 13 & 8 \\
\hline Pigs & - & $10.3-19.8$ \\
\hline Poultry & - & 26 \\
\hline Fallow deer & 11.5 & - \\
\hline
\end{tabular}

${ }^{\text {a }}$ Prevalence on farms in HIV-infected people, before the introduction of the Highly Active Anti-Retroviral Therapy (HAART), ranged from 3.47 to $6.15 \%$ (Angarano et al. 1997; Brandonisio et al. 1999; Giacometti et al. 2000).

Imported giardiosis is also documented in Italy, and an outbreak of G. lamblia infection associated with Entamoeba histolytica was identified in travellers returning from the tropics (Phuket, Thailand; de Lalla et al. 1992).

From a clinical point of view it has been shown that besides diarrhoea, other unexpected symptoms can be detected in patients affected by Giardia. In adult subjects, giardiosis can be associated with chronic atrophic gastritis, intestinal metaplasia of the gastric mucosa and Helicobacter pylori infection (Doglioni et al. 1992), and structural alterations of the retinal pigment epithelium were found to be quite common in paediatric patients with current or past giardiosis (Corsi et al. 1998). Finally, the emerging role of intestinal protozoa in the pathogenesis of Irritable Bowel Syndrome (IBS; Stark et al. 2007) was also confirmed in Italian patients (D'Anchino et al. 2002). In particular, 6.5\% of patients with IBS and dyspepsia presented Giardia infection (Grazioli et al. 2006).

The prevalence of cryptosporidiosis in immunocompetent patients, independent of age, was usually lower than giardiosis and ranged from $1.27 \%$ (Moretti et al. 1988) to $1.7 \%$ (Brandonisio et al. 1996). A low percentage of cryptosporidiosis $(0.84 \%)$ was also found in subjects living in a mental institution (Giacometti et al. 1997). The high prevalence $(5.4 \%)$ recently registered in immunocompetent diarrheic patients in the Tuscany Region (Central Italy) needs to be confirmed because of the conflicting results obtained from microscopy and molecular data (Magi et al. 2006).

A high prevalence of cryptosporidiosis was found in HIV+ patients, before the introduction of the HAART. In fact, a prevalence of $8.33-11 \%$ was registered throughout the Italian Regions (reviewed by Brandonisio et al. 1999); it is interesting that in Southern Italy $21 \%$ of HIV+ subjects with diarrhoea were infected, with a higher incidence in the rainy seasons (Brandonisio et al. 1999).

Evidence of high Cryptosporidium infection rates in Italy derives indirectly from serological investigations. In Central Italy, $5.3 \%$ of healthy people presented antibodies against an oocyst soluble antigen (Gomez Morales et al. 1992); however, these data do not correspond with those obtained in Northern Italy, where an IgG serological response was registered in $62-83 \%$ of blood donors, depending on the antigen used (Frost et al. 2000).

\section{Animal infection}

Giardiosis and cryptosporidiosis in animals have not received as much attention in Italy as other endoparasitoses. 
Although data on these protozoa, as for human infections, date back to the 1960 s, it is only recently that parasitologists and veterinary clinicians have used more reliable epidemiological and diagnostic methodology to reach a better understanding of the spread of these parasites. Table 1 summarises the prevalence in different animal host species.

Companion animals After the first report on the presence of Giardia by De Carneri and Castellino (1964) in 3 out of 31 dogs subjected to postmortem examination from a public animal shelter, surveys carried out subsequently showed that giardiosis prevalence ranges from 3.6 to $9.56 \%$ (Agresti et al. 1977; Piergili-Fioretti and Moretti 1989; Rosso et al. 1989) and can reach $80 \%$ when puppies and/or imported dogs are studied (Traldi and Castiglioni 1993). Since 2000, more statistically significant epidemiological surveys involving privately owned dogs and animal shelter dogs have been carried out and risk factors have been analyzed. Shelter animals constantly presented higher levels of Giardia infection (14-74\%) compared to pet dogs (4.3-19\%), and young animals and subjects with diarrhoea were significantly more affected (Capelli et al. 2003; Bianciardi et al. 2004; Berrilli et al. 2006a; Papini et al. 2005; Paoletti et al. 2006).

As to Cryptosporidium, a clinical case was reported in 1990 in a 6-year-old Pyrennean Mountain dog, whose infection was confirmed by experimental challenge in newborn mice (Traldi 1990). However, no surveys were carried out until very recently when a molecular epidemiological study revealed the presence of Cryptosporidium spp. in 3.3\% of animal shelter and privately owned dogs; here again, shelter dogs and dogs with gastrointestinal symptoms were significantly more affected (Giangaspero et al. 2006).

Regarding Giardia infection in cats, De Carneri and Castellino (1963) found the parasite for the first time in fresh intestinal smears from $5.6 \%$ of 90 autopsied stray cats. The prevalence of $4 \%$ reported by Bianciardi et al. (2004) appears to be underestimated; when larger cat populations are investigated, giardiosis prevalence reaches $15 \%$ (Canestri-Trotti et al. 1990; Papini et al. 2007b) and no risk factor seems to influence such infection (Papini et al. 2007b).

No correlation between infection and immunological status in feline immunodeficiency virus (FIV)-positive cats - except for the amount of cysts excreted-was evidenced by Diaferia et al. (2006); however, such unexpected data require more extensive published reports to draw conclusions. Finally, no data are available for cryptosporidiosis in the feline population.

Farm animals Cryptosporidiosis (Canestri-Trotti et al. 1982; Genchi et al. 1984) and giardiosis (Lalle et al. 2005) affect Italian cattle, although wide prevalence studies are only related to cattle farms; $66.6 \%$ of the Italian farms examined harbour Giardia and 58.3\% Cryptosporidium, and in most cases both protozoans were present (Grana et al. 2006).

Giardiosis does not seem to be a serious problem in a flock of sheep, where a prevalence of only $1.5 \%$ was found in adults (Giangaspero et al. 2005b), but it has been the cause of a malabsorption syndrome, decreased weight gain and impaired feed efficiency in lambs from the same area (Central Italy; Aloiso et al. 2006).

Out of 150 surveyed horses, Giardia has recently been detected in 13\% and Cryptosporidium in $8 \%$ (Veronesi et al. 2006).

It has been known since the 1980s that water buffaloes harbour Cryptosporidium (Canestri-Trotti and Quesada 1983; Canestri-Trotti et al. 1984b), and this was confirmed later (Galiero et al. 1994; Saralli et al. 2001). However, an interesting survey has been published very recently in which Giardia infection has also been reported for the first time. Copro-antigens of $G$. duodenalis and C. parvum occurred in 30 and $24.4 \%$ of farms and in 18.1 and $14.7 \%$ of animals, respectively. Co-infection was present but animals rarely presented diarrhoea (Rinaldi et al. 2006). Finally, in the past, Cryptosporidium has been registered in pigs (Canestri-Trotti et al. 1984a) and also recently (Manfredi et al. 2006) with prevalence levels of up to $19.8 \%$.

Poultry Only one survey is available for poultry in which cryptosporidiosis was detected, with a prevalence of $26 \%$ among roosters and broilers bred in Central Italy and affected by gastro-intestinal symptoms (Piergili-Fioretti et al. 1991). This research came 20 years after a first contribution on the presence of Cryptosporidium in the same species (Mandelli and Valeri 1972).

Wild animals In a survey involving 139 hunted fallow deer (Dama dama), 16 (11.5\%) subjects where found to excrete Giardia cysts (Lalle et al. 2007).

\section{Water contamination}

Drinking, raw and reclaimed water are not routinely monitored for Cryptosporidium and Giardia in Italy; however, studies on the presence of oo/cysts in different kinds of water and wastewater have been recently carried out throughout Italy, and the results are shown in Tables 2, 3 and 4.

Surface water All river water samples appeared to be heavily contaminated because $100 \%$ of examined samples tested positive for Giardia cysts and Cryptosporidium oocysts, with the Giardia load higher than that of Cryptosporidium (Carraro et al. 2000; Briancesco and Bonadonna 2005; Di Benedetto et al. 2005). 
Table 2 Giardia and Cryptosporidium in surface water in Italy

\begin{tabular}{|c|c|c|c|c|c|c|c|c|}
\hline \multirow[t]{2}{*}{ Region } & \multirow[t]{2}{*}{ Years } & \multirow{2}{*}{$\begin{array}{l}\text { Type of } \\
\text { water }\end{array}$} & \multirow{2}{*}{$\begin{array}{l}\text { Number of } \\
\text { samples }\end{array}$} & \multicolumn{2}{|l|}{ Giardia } & \multicolumn{2}{|c|}{ Cryptosporidium } & \multirow[t]{2}{*}{ References } \\
\hline & & & & $\begin{array}{l}\text { Positive } \\
\text { samples (\%) }\end{array}$ & $\begin{array}{l}\text { Mean cyst } \\
\mathrm{N} / \mathrm{L} \pm \mathrm{SD}\end{array}$ & $\begin{array}{l}\text { Positive } \\
\text { samples (\%) }\end{array}$ & $\begin{array}{l}\text { Mean oocyst } \\
\mathrm{N} / \mathrm{L} \pm \mathrm{SD}\end{array}$ & \\
\hline Piedmont & 1997 & River & 22 & $22(100)$ & $1.16 \pm 0.78$ & $22(100)$ & $0.19 \pm 0.08$ & Carraro et al. 2000 \\
\hline Lazio & $\begin{array}{r}2001- \\
2002\end{array}$ & River & 10 & $10(100)$ & $80 \pm 90$ & $10(100)$ & $5 \pm 4$ & $\begin{array}{l}\text { Briancesco and } \\
\text { Bonadonna } 2005\end{array}$ \\
\hline Sicily & $\begin{array}{r}2003- \\
2004\end{array}$ & River & 7 & $7(100)$ & $465 \pm 413$ & $7(100)$ & $9.86 \pm 6.79$ & $\begin{array}{l}\text { Di Benedetto et al. } \\
2005\end{array}$ \\
\hline Sicily & $\begin{array}{r}2003- \\
2004\end{array}$ & Watersheds & 10 & $1(10)$ & $0.12 \pm 0.36$ & $1(10)$ & $0.23 \pm 0.73$ & $\begin{array}{l}\text { Di Benedetto et al. } \\
2005\end{array}$ \\
\hline Lazio & $\begin{array}{r}2001- \\
2002\end{array}$ & Watersheds & 4 & $2(50)$ & $0.006 \pm 0.009$ & 0 & 0 & $\begin{array}{l}\text { Briancesco and } \\
\text { Bonadonna } 2005\end{array}$ \\
\hline Apulia & $\begin{array}{r}2000- \\
2001\end{array}$ & Watersheds & 27 & 0 & 0 & $4(14.8)$ & $0.13 \pm 0.07$ & $\begin{array}{l}\text { Brandonisio et al. } \\
2004\end{array}$ \\
\hline Apulia & $\begin{array}{r}2001- \\
2002\end{array}$ & Watercourse & 7 & $3(42.9)$ & $0.01 \pm 0.02$ & 0 & 0 & $\begin{array}{l}\text { Briancesco and } \\
\text { Bonadonna } 2005\end{array}$ \\
\hline Tuscany & $\begin{array}{r}2003- \\
2004\end{array}$ & Watercourse & 16 & $14(87.5)$ & 2.05 & $10(62.5)$ & 0.19 & Sacco et al. 2006 \\
\hline Lazio & $\begin{array}{r}2001- \\
2002\end{array}$ & Lakes & 3 & $1(33.3)$ & 0.28 & N.I. & N.I. & Di Cave et al. 2005 \\
\hline
\end{tabular}

$N / L$ number of oo/cysts per liter; N.I. Not investigated

In watersheds and watercourses used as source water by the local Municipal Water Companies, Giardia cysts were found in $31 \%$ of samples while Cryptosporidium oocysts were present in 23\% (Brandonisio et al. 2004; Briancesco and Bonadonna 2005; Di Benedetto et al. 2005; Sacco et al. 2006).

Ground water $\mathrm{Oo} / \mathrm{cysts}$ were occasionally found in well water used for agricultural purposes (Briancesco and Bonadonna 2005; Di Benedetto et al. 2005; Lonigro et al. 2006) and especially in wells with a depth of less than $31 \mathrm{~m}$, where faecal bacteria were also found (Di Benedetto et al. 2005).

Drinking and recreational water Neither of the parasites was detected in any of 19 drinking water samples examined (Briancesco and Bonadonna 2005); however, Cryptosporidium and/or Giardia were occasionally detected in swimming pool water (Bonadonna et al. 2004; Briancesco and Bonadonna 2005; Oliveri et al. 2006).

Wastewater In raw sewage and primary effluent, more Giardia cysts were found (up to 108,000/l) than Cryptosporidium oocysts (Carraro et al. 2000; Cacciò et al. 2003; Briancesco and Bonadonna 2005; Di Benedetto et al. 2005).

High concentrations of Giardia cysts, and to a lesser extent of Cryptosporidium oocysts, were also present after secondary treatment of wastewater by activated sludge and sedimentation (Bonadonna et al. 2002; Di Benedetto et al. 2005; Lonigro et al. 2006), while it has been shown that tertiary treatments significantly reduce cyst and oocyst density in wastewater (Brandonisio et al. 2000; Carraro et al. 2000). Final disinfection with chlorine has little effect

Table 3 Giardia and Cryptosporidium in groundwater in Italy

\begin{tabular}{|c|c|c|c|c|c|c|c|}
\hline \multirow[t]{2}{*}{ Region } & \multirow[t]{2}{*}{ Years } & \multirow{2}{*}{$\begin{array}{l}\text { Number of } \\
\text { samples }\end{array}$} & \multicolumn{2}{|l|}{ Giardia } & \multicolumn{2}{|l|}{ Cryptosporidium } & \multirow[t]{2}{*}{ References } \\
\hline & & & Positive samples (\%) & $\begin{array}{l}\text { Mean cyst } \\
\mathrm{N} / \mathrm{L} \pm \mathrm{SD}\end{array}$ & Positive samples (\%) & Mean oocyst N/L & \\
\hline Lazio & $\begin{array}{r}2001- \\
2002\end{array}$ & 14 & 0 & 0 & 0 & 0 & $\begin{array}{l}\text { Briancesco and } \\
\text { Bonadonna } 2005\end{array}$ \\
\hline Apulia & $\begin{array}{r}2004- \\
2005\end{array}$ & 18 & $2(11.1)$ & $0.28 \pm 0.31$ & 0 & 0 & Lonigro et al. 2006 \\
\hline Sicily & $\begin{array}{r}2003- \\
2004\end{array}$ & 14 & $2(14.3)$ & $0.33 \pm 0.25$ & $1(7.1)$ & 1.5 & Di Benedetto et al. 2005 \\
\hline
\end{tabular}


Table 4 Giardia cysts and Cryptosporidium oocysts in wastewater in Italy

\begin{tabular}{|c|c|c|c|c|c|c|c|}
\hline \multirow[t]{2}{*}{ Region } & \multirow{2}{*}{$\begin{array}{l}\text { Type of } \\
\text { wastewater }\end{array}$} & \multirow{2}{*}{$\begin{array}{l}\text { Number of } \\
\text { samples }\end{array}$} & \multicolumn{2}{|l|}{ Giardia } & \multicolumn{2}{|c|}{ Cryptosporidium } & \multirow[t]{2}{*}{ References } \\
\hline & & & $\begin{array}{l}\text { Positive } \\
\text { samples (\%) }\end{array}$ & $\begin{array}{l}\text { Mean/Range } \\
\text { cyst N/L }\end{array}$ & $\begin{array}{l}\text { Positive } \\
\text { samples (\%) }\end{array}$ & $\begin{array}{l}\text { Mean/Range } \\
\text { oocyst N/L }\end{array}$ & \\
\hline Piedmont & $\begin{array}{l}\text { Raw } \\
\text { sewage }\end{array}$ & 3 & $3(100)$ & 54 & $3(100)$ & 4.5 & Carraro et al. 2000 \\
\hline $\begin{array}{l}\text { Lombardy Campania } \\
\text { Sardinia Sicily }\end{array}$ & $\begin{array}{l}\text { Raw } \\
\text { sewage }\end{array}$ & 16 & $16(100)$ & $2,100-42,000$ & $3(18.75)$ & 107 & Cacciò et al. 2003 \\
\hline Lazio & $\begin{array}{l}\text { Raw } \\
\text { sewage }\end{array}$ & 10 & $10(100)$ & $800-7,000$ & $10(100)$ & $0.4-30$ & $\begin{array}{l}\text { Briancesco and } \\
\text { Bonadonna } 2005\end{array}$ \\
\hline Lazio & $\begin{array}{l}\text { Raw } \\
\text { sewage }\end{array}$ & 5 & $5(100)$ & $3,500-34,000$ & N.I. & N.I. & Di Cave et al. 2005 \\
\hline Sicily & $\begin{array}{l}\text { Primary } \\
\text { effluent }\end{array}$ & 8 & $8(100)$ & 108,000 & $8(100)$ & 702 & $\begin{array}{l}\text { Di Benedetto et al. } \\
2005\end{array}$ \\
\hline Sicily & $\begin{array}{l}\text { Secondary } \\
\text { effluent }^{\mathrm{a}}\end{array}$ & 8 & $8(100)$ & 6,160 & $7(87.5)$ & 56.9 & $\begin{array}{l}\text { Di Benedetto et al. } \\
2005\end{array}$ \\
\hline Apulia & $\begin{array}{l}\text { Secondary } \\
\text { effluent }^{\mathrm{a}}\end{array}$ & 4 & $4(100)$ & 1,800 & $2(50)$ & 56 & Lonigro et al. 2006 \\
\hline Apulia & $\begin{array}{l}\text { Tertiary } \\
\text { effluent }^{\mathrm{b}}\end{array}$ & 4 & $4(100)$ & 253 & $4(100)$ & 0.26 & $\begin{array}{l}\text { Brandonisio et al. } \\
2000\end{array}$ \\
\hline Apulia & $\begin{array}{l}\text { Tertiary } \\
\text { effluent }^{\mathrm{c}}\end{array}$ & 4 & $4(100)$ & 6.65 & $4(100)$ & 0.23 & $\begin{array}{l}\text { Brandonisio et al. } \\
2000\end{array}$ \\
\hline Piedmont & $\begin{array}{l}\text { Tertiary } \\
\text { effluent }^{\mathrm{d}}\end{array}$ & 11 & $11(100)$ & 1.4 & $11(100)$ & 0.21 & Carraro et al. 2000 \\
\hline Apulia & $\begin{array}{l}\text { Chlorinated } \\
\text { effluent }\end{array}$ & 14 & $11(78.6)$ & 37.5 & $3(21.4)$ & 0.29 & $\begin{array}{l}\text { Brandonisio et al. } \\
2004\end{array}$ \\
\hline Lazio & $\begin{array}{l}\text { Chlorinated } \\
\text { effluent }\end{array}$ & 6 & $6(100)$ & 60 & $6(100)$ & 30 & $\begin{array}{l}\text { Briancesco and } \\
\text { Bonadonna } 2005\end{array}$ \\
\hline Lazio & $\begin{array}{l}\text { Chlorinated } \\
\text { effluent }\end{array}$ & 2 & $2(100)$ & $31-670$ & N.I & N.I. & Di Cave et al. 2005 \\
\hline Apulia & $\begin{array}{l}\text { Chlorinated } \\
\text { effluent }\end{array}$ & 11 & $9(81.82)$ & 98.85 & $4(36.4)$ & 6.99 & $\begin{array}{l}\text { Brandonisio et al. } \\
2007\end{array}$ \\
\hline Lazio & $\begin{array}{l}\text { UV-treated } \\
\text { effluents }\end{array}$ & 3 & $3(100)$ & 72.33 & N.I & N.I. & Di Cave et al. 2005 \\
\hline Apulia & $\begin{array}{l}\text { UV-treated } \\
\text { effluent }\end{array}$ & 10 & $7(70)$ & 37.8 & $1(10)$ & 2.67 & $\begin{array}{l}\text { Brandonisio et al. } \\
2007\end{array}$ \\
\hline
\end{tabular}

N.I. Not investigated

${ }^{\text {a }}$ Activated sludge

${ }^{\mathrm{b}}$ Chemical flocculation

${ }^{\mathrm{c}}$ Chemical flocculation followed by slow sand filtration

${ }^{\mathrm{d}}$ Chemical dephosphorization and multilayer filtration

on oo/cyst density (Brandonisio et al. 2004; Briancesco and Bonadonna 2005), and chlorine and UV treatments may not influence Giardia cyst viability (Brandonisio et al. 2007).

\section{Food contamination}

In a historical work performed in 1966, samples of Lactuca sativa sold in local markets yielded Giardia cysts (Mastandrea and Micarelli 1968). Almost 50 years later, studies on food have switched to edible shellfish harvested in the sea, lagoons and lakes; Giardia was detected only in Chamelea gallina
(Molini et al. 2004), whereas Cryptosporidium oocysts were detected in C. gallina and Ruditapes philippinarum (Giangaspero et al. 2005a; Molini et al. 2007). Contamination by both parasites was also found in Mytilus galloprovincialis (Sorgi et al. 2007).

\section{Molecular findings}

The occurrence and identification of Giardia and Cryptosporidium isolates in Italy from different sample types are reported in Tables 5, 6, 7 and 8 . 
Table 5 Occurrence and identification of Giardia isolates in Italy from different origins

\begin{tabular}{|c|c|c|c|c|}
\hline Origin & $\begin{array}{l}\text { Number of } \\
\text { isolates }\end{array}$ & PCR target & Genotypes $(\mathrm{N})$ & References \\
\hline Human & 30 & $\beta$-giardin & Ass A (24), Ass B (6) & Cacciò et al. $2002 b$ \\
\hline Human & 37 & $\beta$-giardin & Ass A (17), Ass B (15), Ass (A+B) 5 & Lalle et al. 2005 \\
\hline Human & 11 & $\beta$-giardin & Ass A (5), Ass B (5), Ass (A+B) 1 & Crotti et al. 2005 \\
\hline Human & 42 & $18 \mathrm{~S}$ & Ass A (19), Ass B (13), Ass (A+B) 10 & Berrilli et al. 2006c \\
\hline \multicolumn{5}{|c|}{ Companion animals } \\
\hline Dog & 17 & $18 \mathrm{~S}$ & $\begin{array}{l}\text { Ass A (2), Ass C (11), Ass D (1), Ass }(A+C) 2 \\
\text { Ass (C+D) } 1\end{array}$ & Berrilli et al. 2004 \\
\hline Dog & 21 & $\begin{array}{l}\beta \text {-giardin 18S } \\
\text { GDH }\end{array}$ & Ass A (6), Ass C (1), Ass D (13), Ass (A+D) 1 & Lalle et al. 2005 \\
\hline Dog & 30 & $\beta$-giardin 18S & Ass A (2), Ass C (3), Ass D (25) & Paoletti et al. 2006 \\
\hline Dog & 26 & $18 \mathrm{~S}$ & Ass A (8), Ass C (14), Ass D (4) & Berrilli et al. 2006a \\
\hline Cat & 1 & $18 \mathrm{~S}$ & Ass A (1) & Berrilli et al. 2004 \\
\hline Cat & 1 & $\beta$-giardin GDH & Ass F (1) & Lalle et al. 2005 \\
\hline Cat & 10 & $18 \mathrm{~S}$ & Ass A (10) & Papini et al. 2007a \\
\hline Cat & 8 & Tpi & Ass A (8) & Diaferia et al. 2006 \\
\hline Rabbit & 2 & $18 \mathrm{~S}$ & Ass A (1), Ass $(A+B) 1$ & Berrilli et al. 2006a \\
\hline \multicolumn{5}{|l|}{ Farm Animals } \\
\hline Calf & 3 & $18 \mathrm{~S}$ & Ass E (3) & Berrilli et al. 2004 \\
\hline Calf & 24 & $\beta$-giardin & $\begin{array}{l}\text { Ass A (12), Ass B (5), Ass E (3), Ass (A+B) } 2 \\
\text { Ass (A+E) } 2\end{array}$ & Lalle et al. 2005 \\
\hline Calf & 18 & Tpi $\beta$-giardin & Ass A (7), Ass E (11) & Grana et al. 2006 \\
\hline Sheep & 5 & $\beta$-giardin GDH & Ass A (5) & Giangaspero et al. $2005 \mathrm{~b}$ \\
\hline Sheep & 2 & Tpi & Ass B (2) & Aloisio et al. 2006 \\
\hline \multicolumn{5}{|l|}{ Wild Animals } \\
\hline Fallow deer & 8 & Tpi $\beta$-giardin & Ass A (8) & Lalle et al. 2007 \\
\hline \multicolumn{5}{|l|}{ Water } \\
\hline Wastewater & 16 & $\beta$-giardin & Ass A (8), Ass $(A+B) 8$ & Cacciò et al. 2003 \\
\hline Wastewater & 10 & $18 \mathrm{~S}$ & Ass A (10) & Di Cave et al. 2005 \\
\hline Wastewater & 6 & $18 \mathrm{~S}$ & Ass A (5), Ass B (1) & Lonigro et al. 2006 \\
\hline Wastewater & 14 & 18S Tpi & Ass A (10), Ass B (1) Ass A or B (3) ${ }^{\mathrm{a}}$ & Brandonisio et al. 2007 \\
\hline $\begin{array}{l}\text { Surface } \\
\text { water }\end{array}$ & 3 & $18 \mathrm{~S}$ & Ass A (3) & Di Cave et al. 2005 \\
\hline Watercourses & 7 & 18S Tpi & Ass A (6), Ass B (1) & Brandonisio et al. 2007 \\
\hline
\end{tabular}

${ }^{\mathrm{a}}$ Incoherent results given by using two different loci

Humans One hundred and twenty different Giardia DNA samples from human stools have been genotyped, 54.16\% displayed Giardia Assemblage A, 32.5\% Assemblage B and 13\% harbour mixed Assemblage A/B. Giardia intraAssemblage variations have also been detected in Italy for both Assemblages, within Assemblage A, seven subgenotypes (referred to as ' $\mathrm{A} 1-\mathrm{A} 7$ ') were identified and three subgenotypes (B1, B3 and B4) within Assemblage B (Cacciò et al. 2002b; Lalle et al. 2005; Crotti et al. 2005). Out of ten genotyped Cryptosporidium isolates, nine isolates were identified as C. parvum, and in one case, C. felis was detected (Cacciò et al. 2000, 2001, 2002a).

Companion animals Ninety-four Giardia isolates from dogs have been molecularly identified, $76.5 \%$ belonged to species-specific Assemblages (C and D) while 19\% belonged to Assemblage A and 4\% to mixed Assemblages. Twenty isolates have been characterised from cats in Northern and Central Italy and all belonged to Assemblage A (Berrilli et al. 2004; Papini et al. 2007a; Diaferia et al. 2006) except for one belonging to Assemblage F (Lalle et al. 2005). Two isolates from rabbits harboured Assemblage A and mixed Assemblages (A + B; Berrilli et al. 2006a).

As to Cryptosporidium, nine isolates have been genotyped from dogs and one from hamsters, C. canis was detected in dogs while, more notably, both animal species harboured C. parvum (Giangaspero et al. 2006; Cencioni 2005; Berrilli et al. 2006a).

Farm animals Giardia and Cryptosporidium genotypes/ species have been detected in cattle, sheep, goats and pigs for a total of 52 and 68 isolates typed, respectively. 
Table 6 Giardia genotypes from different sample types in Italy

\begin{tabular}{|c|c|c|c|c|c|c|c|c|c|}
\hline Origin & $\begin{array}{l}\text { Total } \\
\text { samples }\end{array}$ & $\begin{array}{l}\text { Ass A } \\
(\%)\end{array}$ & $\begin{array}{l}\text { Ass B } \\
(\%)\end{array}$ & $\begin{array}{l}\text { Ass } A \text { or } \mathrm{B}^{\mathrm{a}} \\
(\%)\end{array}$ & $\begin{array}{l}\text { Ass C } \\
(\%)\end{array}$ & $\begin{array}{l}\text { Ass D } \\
(\%)\end{array}$ & $\begin{array}{l}\text { Ass E } \\
(\%)\end{array}$ & $\begin{array}{l}\text { Ass F } \\
(\%)\end{array}$ & Mixed assemblage (\%) \\
\hline Human & 120 & $65(54.2)$ & $39(32.5)$ & & - & - & - & - & $(A+B) 16(13,3)$ \\
\hline Dog & 94 & $18(19.1)$ & - & & $29(31.0)$ & $43(45.7)$ & - & - & $\begin{array}{l}(\mathrm{A}+\mathrm{C}) 2(\mathrm{C}+\mathrm{D}) 1(\mathrm{~A}+\mathrm{D}) \\
1(4.2)\end{array}$ \\
\hline Cat & 20 & $19(95.5)$ & - & & - & - & - & $1(0.5)$ & - \\
\hline Rabbit & 2 & $1(50.0)$ & - & & - & - & - & - & $(\mathrm{A}+\mathrm{B}) 1(50.0)$ \\
\hline Calf & 45 & $19(42.2)$ & $5(11.1)$ & & - & - & $17(37.8)$ & - & $(\mathrm{A}+\mathrm{B}) 2(\mathrm{~A}+\mathrm{E}) 2(8.9)$ \\
\hline Sheep & 7 & $5(71.4)$ & $2(28.6)$ & & - & - & - & - & - \\
\hline $\begin{array}{c}\text { Fallow } \\
\text { deer }\end{array}$ & 8 & $8(100)$ & - & & - & - & - & - & - \\
\hline Water & 56 & $42(75.0)$ & $3(5.35)$ & $3(5.35)$ & - & - & - & - & $(\mathrm{A}+\mathrm{B}) 8(14.2)$ \\
\hline TOT & 352 & $177(50.3)$ & $49(13.9)$ & $3(0.8)$ & $29(8.2)$ & $43(12.2)$ & $17(4.8)$ & $1(0.3)$ & $33(9.3)$ \\
\hline
\end{tabular}

${ }^{\mathrm{a}}$ Incoherent results given by using two different loci

Forty-five Giardia isolates from cattle were mainly identified as Assemblage A (42\%) followed by the speciesspecific Assemblage E (38\%), Assemblage B (11\%) and mixed Assemblages (9\%; Berrilli et al. 2004; Lalle et al. 2005; Grana et al. 2006); in these isolates, a number of subgenotype (A1, A2, A3, A4, B3 and the new subgenotypes B5 and B6) were identified (Lalle et al. 2005).

Seven isolates have been typed in sheep, and Assemblages A1 and B have been detected (Giangaspero et al. 2005b; Aloisio et al. 2006).

As regards Cryptosporidium, only C. parvum isolates were identified in all farm animals (Cacciò et al. 2000, 2001; Cencioni 2005; Grana et al. 2006).
Finally, C. suis and the pig genotype II were identified in 12 faecal pools from pigs (Manfredi et al. 2006).

Wild animals Eight faecal samples collected from fallow deer were all identified as a new unique subtype within Assemblage A (Lalle et al. 2007).

Water and wastewater Molecular typing of Giardia and Cryptosporidium in Italy was performed in wastewater (Cacciò et al. 2003; Di Cave et al. 2005; Lonigro et al. 2006) and in surface water (Di Cave et al. 2005). Although this referred to a limited number of Giardia isolates (57), most (up to $74 \%$ ) belonged to Assemblage A, while

Table 7 Occurrence and identification of Cryptosporidium isolates from different origins in Italy

\begin{tabular}{lclll}
\hline Origin & $\begin{array}{c}\text { Number of } \\
\text { isolates }\end{array}$ & PCR target & Species/Genotypes (N) & References \\
\hline Human & 9 & Microsatellite & C. parvum (9) & Cacciò et al. 2000, 2001 \\
Human & 1 & SSUrRNA COWP & C. felis (1) & Cacciò et al. 2002a \\
Dog & 1 & COWP & C.canis (1) & Cencioni 2005 \\
Dog & 8 & COWP & C.canis (1) C.parvum (7) & Giangaspero et al. 2006 \\
Hamster & 1 & COWP & C.parvum (1) & Berrilli et al. 2006a \\
Calf & 29 & Microsatellite & C.parvum (29) & Cacciò et al. 2000, 2001 \\
Calf & 3 & COWP & C.parvum (3) & Cencioni 2005 \\
Calf & 15 & COWP 18S rDNA & C.parvum (15) & Grana et al. 2006 \\
Kid & 11 & Microsatellite & C.parvum (11) & Cacciò et al. 2000, 2001 \\
Lamb & 10 & Microsatellite & C.parvum (10) & Cacciò et al. 2000, 2001 \\
Pig & 12 & 18S rDNA & C. suis (8) pig genotype II (4) & Manfredi et al. 2006 \\
Shellfish (C. gallina) & $2^{\text {a }}$ & COWP & C.parvum (2) & Traversa et al. 2004; Giangaspero et al. \\
& & & & 2005a \\
Shellfish & $7^{\text {a }}$ & COWP & C.parvum (6) C.hominis (1) & Molini et al. 2007 \\
(R.philippinarum) & & COWP & C.parvum (2) & Lonigro et al. 2006 \\
Wastewater & 2 & COWP & C.parvum (1) & Brandonisio et al. 2007 \\
Watercourses & 1 & &
\end{tabular}

${ }^{\mathrm{a}}$ The number refers to pooled samples. 
Table 8 Cryptosporidium species/genotypes of different origins in Italy

\begin{tabular}{|c|c|c|c|c|c|c|c|}
\hline Origin & Total samples & C. parvum (\%) & C. hominis (\%) & C. felis (\%) & C.canis $(\%)$ & C. suis $(\%)$ & Pig genotype II (\%) \\
\hline Human & 10 & $9(90.0)$ & - & $1(10.0)$ & & - & - \\
\hline Dog & 9 & $7(77.8)$ & - & - & $2(22.2)$ & - & - \\
\hline Hamster & 1 & $1(100)$ & - & - & - & - & - \\
\hline Calf & 47 & $47(100)$ & - & - & - & - & - \\
\hline Kid & 11 & $11(100)$ & - & - & - & - & - \\
\hline Lamb & 10 & $10(100)$ & - & - & - & - & - \\
\hline Pig & 12 & - & - & - & - & $8(66.7)$ & $4(33.3)$ \\
\hline Shellfish & $9^{\mathrm{a}}$ & $8(88.9)$ & $1(11.1)$ & - & - & - & - \\
\hline Water & 3 & $3(100)$ & - & - & - & - & - \\
\hline TOT & 112 & $96(85.7)$ & $1(0.8)$ & $1(0.8)$ & $2(1.7)$ & $8(7.1)$ & $4(3.5)$ \\
\hline
\end{tabular}

${ }^{\mathrm{a}}$ The number refers to pooled samples.

Assemblage B (7\%) was poorly represented and mixed Assemblages $(\mathrm{A}+\mathrm{B})$ were detected in $16 \%$. Interestingly, incoherent results were obtained in three cases when two different loci targets were used (Brandonisio et al. 2007). Two isolates of Cryptosporidium from secondary-treated wastewater (Lonigro et al. 2006) and one from a watercourse were identified as C. parvum (Brandonisio et al. 2007).

Food Sequencing analysis was carried out on samples from $C$. gallina and $R$. philippinarum clams harvested along the Adriatic coast; C. parvum was found in both clam species, and $C$. hominis was also detected in $R$. philippinarum (Giangaspero et al. 2005a; Molini et al. 2007).

\section{Discussion}

Data on the prevalence of giardiosis and cryptosporidiosis in humans, animals and the environment vary greatly in Italy according to geographical location, the population studied and diagnostic methods used.

Well-standardised procedures are available for carrying out laboratory diagnosis of human giardiosis/cryptosporidiosis (http://www.dpd.cdc.gov/dpdx/HTML/Giardiasis.htm; http://www.dpd.cdc.gov/dpdx/HTML/Cryptosporidiosis. htm; Cacciò and Pozio 2006; Savioli et al. 2006), but special acid-fast staining or immunodiagnostic techniques are needed for Cryptosporidium in particular. Because of its greater sensitivity and specificity, immunofluorescence microscopy is the method of choice in the detection of this coccidian parasite, followed closely by enzyme immunoassays (Savioli et al. 2006). Unfortunately, these methods are not routinely performed in parasitological stool examination, so that Cryptosporidium and other intestinal coccidians may often be missed in humans.

In animals, most prevalence investigations in the Italian arena have principally been based on the detection of oo/ cysts by light microscopy followed by immunofluorescence or enzyme-linked immunosorbent assay (ELISA) tests; however, the application of immunoassays in animals for epidemiological survey is still a matter for debate. This due to the fact that the test has not yet been approved for use in veterinary medicine, and, more importantly, the need to confirm positive ELISA results by (repeated) faecal examination using other detection methods has been demonstrated (Cirak and Bauer 2004).

Techniques based on DNA detection such as polymerase chain reaction (PCR) assays are too expensive for prevalence studies in humans and animals and thus are not widely applied in Italy. Different methods have also been used to identify protozoa in water-especially in wastewater-and consequently, the results are often difficult to compare. In Italy, methods suggested by the National Institute of Health (Annali ISTISAN 2003) are mainly used for detecting Giardia and Cryptosporidium in raw and drinking water (concentration by filtration capsules or a compressed foam filter system, followed by purification by Percoll-sucrose or immunomagnetic separation and identification by immunofluorescent antibodies). Different methods were used for wastewater because there are no well-standardised protocols available.

While the prevalence of human giardiosis and cryptosporidiosis evaluated by stool examination overlaps that of other industrialised countries (de Wit et al. 2001; Ali and Hill 2003; Horman et al. 2004; Fayer 2004; Joachim 2004), the seroprevalence of cryptosporidiosis seems to be unexpectely high in asymptomatic subjects, at least in a geographically limited area of Northern Italy (Frost et al. 2000) when compared to other countries (Frost et al. 2004; Fayer et al. 2004). If confirmed on larger subject numbers, this serological condition may suggest a lower risk of symptomatic cryptosporidiosis in that area, because of recent seroepidemiological studies showing that people with high anti-Cryptosporidium antibody levels have less risk of diarrhoeal or gastrointestinal illness (Frost et al. 2005a; Frost et al. 2005b). 
In recent years, HAART has drastically reduced the prevalence of cryptosporidiosis in HIV+ patients in Italy (Conti et al. 2000; Manfredi 2000; Maggi et al. 2001), also due to direct effects on Cryptosporidium of aspartyl protease inhibitors of the HIV, which interfere with the life cycle of the parasite (Mele et al. 2003). However, the presence of such infections must be monitored in HIV+ patients because relapses have been recorded in patients who interrupted the combined anti-retroviral therapy (Maggi et al. 2000).

No epidemiological surveys are available for immigrant populations; however, this may allow for some considerations. Immigration is on the increase in Italy, an impressive new phenomenon, and most immigrants arrive from Eastern European Countries and Albania in primis (ISTAT 2005). From an epidemiological point of view, this sociological event may facilitate the spread of giardiosis, considering that $G$. duodenalis prevalence has recently been found to be as high as $12.27 \%$ in healthy (Spinelli et al. 2006) and symptomatic (Berrilli et al. 2006b) Albanians. In addition, foreign travel is confirmed as a risk factor for giardiosis (Faustini et al. 2006).

Among animals, dogs are the most studied species for giardiosis in Italy and prevalence appears to be of concern mainly in dogs that are well cared for (up to 19\%) compared to other European countries (Barr and Bowman 1994; Borkovcova 2003).

Interesting data are emerging from cats because the high prevalence level $(15.8 \%)$ registered in Italy confirms that cats are not poor or inadequate hosts nor limited reservoirs as previously reported (Hill et al. 2000; McGlade et al. 2003; Vasilopulos et al. 2006).

Besides the confirmed risk factors of young age and confinement, diarrhoea and Giardia seems to be closely associated in dogs. In addition, the possibility that dogs fed on commercial wet food are more susceptible to Giardia infection (Papini et al. 2005) is an intriguing point for future investigation.

Thanks to more incisive information, it seems that in the last few years greater attention has been paid by both Italian veterinary practitioners and owners to screening and treating puppies for giardiosis; this is also due to the availability of safe and effective treatment (Giangaspero et al. 2002). The Italian epidemiological data regarding Cryptosporidium prevalence are too scarce to allow a conclusion about pets and farm animals, and clinicians in the country display no such awareness. Further limits are created by the difficulty of detecting Cryptosporidium in clinical laboratory conditions and the lack of new and effective molecules for control (Benbow et al. 1998; Yu and Choi 2000).

As regards environmental dispersion of these parasites, although wastewater, surface, ground and recreational water were contaminated by oo/cysts throughout Italy, drinking water was not. However, Giardia and Cryptosporidium testing is not routinely performed in drinking water, raw water and reclaimed water, in accordance with the European Directive 91/271/CEE on the treatment of municipal wastewater, Italian Law (Decree 152/1999) and the European Directive 98/83/CE on the quality of water intended for human consumption. Although a high concentration of Giardia has been detected in water, only one outbreak of waterborne cryptosporidiosis has been reported in Italy. It occurred in January and February 1995, and involved 294 out of 1731 members of a community for the rehabilitation of drug users. The rate of clinical cryptosporidiosis was $13.6 \%$ among HIV negative subjects and $30.7 \%$ among HIV positive subjects. Cryptosporidium oocysts were found in the sandy sediment collected from the bottom of two water storage tanks serving the community (Pozio et al. 1997).

It has also been shown in Italy that disinfection of wastewater is not always sufficient to ensure good oo/cysts removal and that the removal capacity varied greatly depending on the technology used in the treatment plants. These findings suggest the need for monitoring the presence of oo/cysts, especially in wastewater for reuse, and the necessity of insisting on advanced tertiary treatment for producing reclaimed water with no negative impact on public health. In this respect, recent work carried out in Southern Italy has demonstrated that a new wastewater tertiary treatment system based on membrane ultrafiltration is useful in removing pathogenic protozoa from secondarytreated municipal wastewater without chlorine disinfection (Lonigro et al. 2006).

An important point is that contaminated faeces and rainfall-initiated runoff and wastewater carrying protozoa oo/cysts run into rivers, estuaries and coastal waters, thus contaminating sea and shellfish. The identification of Giardia and Cryptosporidium in two clam species (R. philippinarum and C. gallina; Giangaspero et al. 2005a; Molini et al. 2007) provided evidence of the high level of faecal pollution along the Italian Adriatic coast and above all in the Venice Lagoon, where contaminated shellfish were found in different seasons (Molini et al. 2007). The overall prevalence of contamination by Giardia and Cryptosporidium in harvested Italian shellfish is still unknown, and considering that (a) each shellfish may transport more than $10^{3}$ oocysts (Gomez-Couso et al. 2003), (b) shellfish depuration processes do not totally remove Cryptosporidium and moreover, that (c) usual shellfish cooking methods (steaming) do not eliminate the presence and the infectivity of $C$. parvum oocysts, as demonstrated in M. galloprovincialis by Gomez-Couso et al. (2006), such findings are a matter for concern in Italy. Shellfish represent more than $10 \%$ of Italian fishing products (Mattei and Pellizzato 1997) and are a major food 
source in the country, where large quantities are typically eaten raw or uncooked and sometimes even sold illegally.

Molecular characterization of Giardia and Cryptosporidium isolates appears important for providing accurate information about host specificity, zoonotic potential and possible routes of transmission.

Different DNA targets are usually utilised to detect Giardia in environmental and faecal samples (see Cacciò et al. 2005 and Smith et al. 2006), and the choice of a specific molecular typing method mainly depends on the purpose of the study. However, analysis should be carried out on the basis of PCR targets that have been used successfully in epidemiological or taxonomic studies and for which the sequences are multicentre validated.

In Italy, for molecular identification of Giardia cysts and Cryptosporidium oocysts from human/animals stools and environmental samples, most of the common loci have been used (Tables 5 and 7) with different assays; a PCR-RFLP targeting the $\beta$-giardin gene (Cacciò et al. 2002b) was developed for the rapid discrimination of Assemblages and more recently a fluorescence resonance energy transfer real-time PCR has been developed to detect and quantify $G$. duodenalis cysts (Berrilli et al. 2006c). Cryptosporidium oocyst wall protein (COWP) has been the most targeted gene for Cryptosporidium and a semi-nested PCR has been performed (Traversa et al. 2004; Giangaspero et al. 2005a) as well as mini- and microsatellites for subgenotyping (Cacciò et al. 2000, 2001).

When two loci have been used simultaneously for Giardia (Brandonisio et al. 2007), the contradictory results obtained open up serious discussion about the reliability of genotyping based on only one gene target.

A total of 353 Giardia isolates of various origin have been genotyped in Italy, and zoonotic Assemblage A seems to be the most widespread - with the highest levels in humans and livestock (50\%), followed by Assemblage B (14\%) found especially in humans but also in cattle, while mixed Assemblages were present in 9\%.

The wider distribution in Italy of Assemblage A in humans seems to be in contrast with the results reported from other countries, where Assemblage B appears more common, as reviewed by (Cacciò et al. 2005), who have listed genotyping of 1,438 human faecal samples from 14 different countries and reported that $69 \%$ of those samples belong to Assemblage B and $26 \%$ to Assemblage A. However, although consistent dominance of Assemblage B seems to be unequivocal with regard to England and Wales - where most of the samples (1206) come from-in all other surveyed countries, such high Assemblage B prevalence appears to be strongly biased by undersampling. In fact, in Uganda, Korea and Ohio, $100 \%$ Assemblage A is reported, but the analyses were conducted on only 3,5 and 14 isolates, respectively.

Data from Italy are based on 120 human isolates tested until now, so that data on the higher prevalence of
Assemblage A over Assemblage B appear to be more significant and reliable. Considering that the greatest zoonotic risk is from Assemblage A and that Assemblage B represents a lower risk because it appears to be mainly human transmitted, we assume the existence of different transmission cycles within diverse geographical regions. In animals, both zoonotic and non-zoonotic Assemblages were found to be associated with Giardia infections. Interestingly, while dogs harbour more host specific Assemblages, other species such as cats and cattle harbour a greater number of zoonotic Assemblages, thus acting as a potential source of infection for humans.

Subgenotypes of Giardia Assemblages A and B have also been detected in humans and animals in Italy; however, the epidemiological significance of these subgenotypes is still obscure (see Hunter and Thompson 2005).

Regarding Cryptosporidium, 112 human, animal and environmental samples have been genotyped and the zoonotic species $C$. parvum was the most frequently detected $(85.7 \%)$, mainly in livestock but also in dogs, water, wastewater and shellfish. This widespread presence of C. parvum poses an unquestionably high level of risk of disease for humans from these reservoirs in Italy.

\section{Conclusion and future perspectives}

Since 2002, after a worldwide shared agreement to map the epidemiological situation and the risks related to Giardia and Cryptosporidium infections, Italian research has added more information about prevalence and molecular data from different origins and areas of the country and contributed to the development of reliable molecular assays.

Data available in Italy indicate widespread faecal contamination by Giardia and Cryptosporidium from land to sea. Giardia and/or Cryptosporidium have been detected in humans, companion animals, sheep, cattle and water buffaloes, in wastewater, surface water, and also in vegetables and shellfish. Interestingly, many species of animals harbour more zoonotic species/genotypes (Assemblage A G. duodenalis and C. parvum) than species-specific ones.

It seems that in Italy the role of farm animals can be significant for human infection, due to the higher circulation of Assemblage A of Giardia and C. parvum among humans and animals, and among pet cats.

For cryptosporidiosis, analysis of outbreaks and casecontrol studies coupled with genotyping data in other geographical areas have confirmed the public health significance of zoonotic transmission, especially from cattle to humans through direct contact with infected cattle or transmission through drinking water (Hunter and Thompson 2005). 
For Giardia, even if some genotypes can circulate between humans and dogs, the higher prevalence of host specific genotypes in Italy may suggest that zoonotic transmission is unlikely to occur, except in particular socio-cultural conditions such as those registered in a localised endemic focus in India (Traub et al. 2004).

In addition, although still referred to a limited number of collecting sites, the constant occurrence of zoonotic species/ Assemblage in wastewaster and surface water suggests a high circulation of Giardia and Cryptosporidium in the environment and the potential risk associated with the reuse of wastewater.

In Italy, while the prevalence of sporadic human giardiosis and cryptosporidiosis is similar to other industrialised countries, only one outbreak of waterborne cryptosporidiosis has been documented. This could be because, in comparison to other countries such as England and Wales, Italy uses more water drawn from aquifers than surface water as a source for drinking water treatment plants, and another reason may be the very high consumption of mineral water (among the highest in Europe). However, the possibility that small outbreaks have not been detected because of failures in the surveillance system cannot be ruled out.

It will be possible to draw conclusions in terms of public health when more consistent data on molecular epidemiology (i.e. prevalence studies on a larger number of animal species coupled with genotyping, especially from localised transmission foci) become available in our country. In addition, the quality of current genotyping tools - mostly based on analysis of single genetic loci-should be further improved. At least two loci should be investigated to obtain the sufficiently reliable information, and one locus should be $18 \mathrm{~S}$ ribosomal DNA for both parasites (Smith et al. 2006). A multi-locus approach to characterization of Giardia and Cryptosporidium isolates appears essential to provide a broad consensus on their taxonomic status. However, caution is necessary in describing species and intraspecific intragenotype variants or genotypes; this represents an important field for further investigation.

\section{References}

Agresti A, D'Ambrosio G, Gravino E (1977) La Giardiasi del cane. Indagine epizoologica ed osservazioni cliniche. Acta Med Vet 23:175-182

Ali SA, Hill DR (2003) Giardia intestinalis. Curr Opin Infect Dis $16: 453-460$

Aloisio F, Filippini G, Antenucci P, Lepri E, Pezzotti G, Cacciò SM, Pozio E (2006) Severe weight loss in lambs infected with Giardia duodenalis assemblage B. Vet Parasitol 142:154-158

Angarano G, Maggi P, Di Bari MA, Larocca AM, Congedo P, Di Bari C, Brandonisio O, Chiodo F (1997) Giardiasis in HIV: a possible role in patients with severe immune deficiency. Eur J Epidemiol 13:485-487
Ballone E, Fazii P, Riario Sforza G, Scassa E, Di Nicola M, Ippolito N, Di Mascio C, Schioppa F (2001) Survey on giardiasis propagation in Pescara. Ann Ig 13:111-120

Barr SC, Bowman DD (1994) Giardiasis in dogs and in cats. Comp Contin Educ Pract Veter 16:603-610

Benbow JW, Bernberg EL, Korda A, Mead JR (1998) Synthesis and evaluation of dinitroanilines for treatment of cryptosporidiosis. Antimicrob Agents Chemother 42:339-343

Berrilli F, Di Cave D, De Liberato C, Franco A, Scaramozzino P, Orecchia P (2004) Genotype characterization of Giardia duodenalis isolates from domestic and farm animals by SSUrRNA gene sequencing. Vet Parasitol 122:193-199

Berrilli F, Di Cave D, D’Orazi C, Dongiovanni B, Scaramozzino P, Scholl F, De Liberato C (2006a) Giardia e Cryptosporidium in kennel dogs, pet rabbits and hamsters from Rome (Italy). Parassitologia 48:261

Berrilli F, Di Cave D, D’Orazi C, Orecchia P, Xhelilaj L, Bejko D, Caca P, Bebeci D, Cenko F, Donia D, Divizia M (2006b) Prevalence and genotyping of human isolates of Giardia duodenalis from Albania. Parasitol Int 4:295-297

Berrilli F, Di Cave D, D’Orazi C, Valentini A, Iori R, Bernardini S (2006c) Development of a FRET real-time polymerase chain reaction assay for quantitative detection of Giardia duodenalis. Parassitologia 48:172

Bianciardi P, Papini R, Giuliani G, Cardini G (2004) Prevalence of Giardia antigen in stool samples from dogs and cats. Rev Med Vet 155:417-421

Bonadonna L, Briancesco R, Ottaviani M, Veschetti E (2002) Occurrence of Cryptosporidium oocysts in sewage effluents and correlation with microbial, chemical and physical water variables. Environ Monit Assess 75:241-252

Bonadonna L, Briancesco R, Magini V, Orsini M, Romano-Spica V (2004) A preliminary investigation on the occurrence of protozoa in swimming pools in Italy. Ann Ig 16:709-719

Borkovcova M (2003). Prevalence of intestinal parasites of dogs in rural areas of South Moravia (Czech Republic). Helminthologia 40:141-146

Brandonisio O, Marangi A, Panaro MA, Marzio R, Natalicchio MI, Zizzadoro P, De Santis U (1996) Prevalence of Cryptosporidium in children with enteritis in southern Italy. Eur J Epidemiol 12:187-190

Brandonisio O, Maggi P, Panaro MA, Lisi S, Andriola A, Acquafredda A, Angarano G (1999) Intestinal protozoa in HIV-infected patients in Apulia, South Italy. Epidemiol Infect 123:457-462

Brandonisio O, Portincasa F, Torchetti G, Lacarpia N, Rizzi A, Fumarola L, Donadio F, Carnimeo D (2000) Giardia and Cryptosporidium in water: evaluation of two concentration methods and occurrence in wastewater. Parassitologia 42:205-209

Brandonisio O, Fumarola L, Spinelli R, Donadio F, Montemurro P, Portincasa F (2004) Giardia e Cryptosporidium spp.: rassegna critica e monitoraggio in acque superficiali e reflue. Ig Mod 122:137-160

Brandonisio O, Lonigro A, Lacasella V, Cavallo P, Berrilli F, Di Cave D, Cirillo R, Marangi M, Giangaspero A (2007) Cryptosporidium and Giardia in treated municipal wastewater and water courses flowing into the Varano Lagoon (Italy) and in shellfish harvested in the same environment. Proc. II Int Congress on Giardia and Cryptosporidium, Morales, Mexico 13-18 May, 2007

Briancesco R, Bonadonna L (2005) An Italian study on Cryptosporidium and Giardia in wastewater, fresh water and treated water. Environ Monit Assess 104:445-457

Cacciò SM, Pozio E (2006) Advances in the epidemiology, diagnosis and treatment of cryptosporidiosis. Expert Rev Anti Infect Ther 4:429-443

Cacciò S, Homan W, Camilli R, Traldi G, Kortbeek T, Pozio E (2000) A microsatellite marker reveals population heterogeneity within 
human and animal genotypes of Cryptosporidium parvum. Parasitology 120:237-244

Cacciò S, Spano F, Pozio E (2001) Large sequence variation at two microsatellite loci among zoonotic (genotype C) isolates of Cryptosporidium parvum. Int J Parasitol 31:1082-1086

Cacciò S, Pinter E, Fantini R, Mezzaroma I, Pozio E (2002a) Human infection with Cryptosporidium felis: case report and literature review. Emerg Infect Dis 8:85-88

Cacciò SM, De Giacomo M, Pozio E (2002b) Sequence analysis of the beta-giardin gene and development of a polymerase chain reaction-restriction fragment length polymorphism assay to genotype Giardia duodenalis cysts from human faecal samples. Int J Parasitol 32:1023-1030

Cacciò SM, De Giacomo M, Aulicino FA, Pozio E (2003) Giardia cysts in wastewater treatment plants in Italy. Appl Environ Microbiol 69:3393-3398

Cacciò SM, Thompson RC, McLauchlin J, Smith HV (2005) Unravelling Cryptosporidium and Giardia epidemiology. Trends Parasitol 21:430-437

Canestri-Trotti G, Gramenzi F, Foglini A, Tognato G (1982) Prima segnalazione di protozoi del genere Cryptosporidium in vitelli in Italia. Atti Soc Ital Buiat 14:289-292

Canestri-Trotti G, Quesada A (1983) Primo reperto di Cryptosporidium sp. in bufali italiani (Bubalus bubalis). Atti Soc Ital Sci Vet 3:737

Canestri-Trotti G, Pampiglione S, Visconti S (1984a). Cryptosporidium sp. e Isospora suis nei suino in Italia. Parassitologia 26:299_ 304

Canestri-Trotti G, Quesada A, Visconti S (1984b). Ricerche sulla fauna protozoaria intestinale del bufalo (Bubalus bubalis). Atti Soc Ital Buiat 16:443

Canestri-Trotti G, Corradini L, Visconti S (1990) Indagine parassitologica in un rifugio per gatti a Ferrara. Parassitologia 32:42-43

Capelli G, Paoletti B, Iorio R, Frangipane Di Regalbono A, Pietrobelli M, Bianciardi P, Giangaspero A (2003) Prevalence of Giardia spp. in dogs and humans in Northern and Central Italy. Parasitol Res 90:154-155

Caprioli A, Pezzella C, Morelli R, Giammanco A, Arista S, Crotti D, Facchini M, Guglielmetti P, Piersimoni C, Luzzi I (1996) Enteropathogens associated with childhood diarrhea in Italy. Pediatr Infect Dis J 15:876-883

Carraro E, Fea E, Salva S, Gilli G (2000) Impact of a wastewater treatment plant on Cryptosporidium oocysts and Giardia cysts occurring in surface water. Water Sci Technol 41:31-37

Cencioni V (2005) Applicazione della PCR per la diagnosi e la tipizzazione di Cryptosporidium spp. in isolati umani e animali. Degree Thesis AA 2005-2006

Cevenini R, Varoli O, Rumpianesi F, Mazzaracchio R, Nanetti A, La Placa M (1985) A two-year longitudinal study on the etiology of acute diarrhea in young children in Northern Italy. Microbiologica 8:51-58

Cirak VY, Bauer C (2004) Comparison of conventional coproscopical methods and commercial coproantigen ELISA kits for the detection of Giardia and Cryptosporidium infections in dogs and cats. Berl. Munch Tierarztl Wonchenschr 117:410-413

Conti S, Masocco M, Pezzotti P, Toccaceli V, Vichi M, Boros S, Urciuoli R, Valdarchi C, Rezza G (2000). Differential impact of combined antiretroviral therapy on the survival of Italian patients with specific AIDS-defining illnesses. J Acquir Immune Defic Syndr 25:451-458

Corsi A, Nucci C, Knafelz D, Bulgarini D, Di Iorio L, Polito A, De Risi F, Ardenti Morini F, Paone FM (1998) Ocular changes associated with Giardia lamblia infection in children. $\mathrm{Br} \mathrm{J}$ Ophthalmol 82:59-62

Crotti D, D'Annibale ML, Fonzo G, Lalle M, Cacciò SM, Pozio E (2005) Dientamoeba fragilis is more prevalent than Giardia duodenalis in children and adults attending a day care centre in Central Italy. Parasite 12:165-170

D'Anchino M, Orlando D, De Feudis L (2002) Giardia lamblia infections become clinically evident by eliciting symptoms of irritable bowel syndrome. J Infect 45:169-172

De Carneri I, Castellino S (1963) Bassa incidenza di Giardia cati Deschiens 1925 e assenza di protozoi orali nei gatti a Milano. Riv Parassitol 24:1-4

De Carneri D, Castellino S (1964) Entamoeba canibuccalis, Trichomonas canistomae, Giardia canis nei cani a Milano. Clin Vet 87:193-196

de Lalla F, Rinaldi E, Santoro D, Nicolin R, Tramarin A (1992) Outbreak of Entamoeba histolytica and Giardia lamblia infections in travellers returning from the tropics. Infection 20:78-82

De Riu GC, Vitale A, Margarita G (1967) On the distribution of Lamblia intestinalis in a population group in the province of Caserta. Ig Mod 60:270-275

de Wit MA, Koopmans MP, Kortbeek LM, van Leeuwen NJ, Vinje J, van Duynhoven YT (2001). Etiology of gastroenteritis in sentinel general practices in The Netherlands. Clin Infect Dis 33:280-288

Di Benedetto MA, Di Piazza F, Maida CM, Firenze A, Oliveri R (2005) Occurrence of Giardia and Cryptosporidium in wastewater, surface water and ground water samples in Palermo (Sicily). Ann Ig 17:367-375

Di Cave D, Berrilli F, Orecchia P, Mecozzi B, Donia D, Bonadonna L, Briancesco R, Divizia M (2005) Detection and genotypic characterization of Giardia cysts in water samples. Ig Sanità Publica 61:9-16

Diaferia M, Cacciò SM, Veronesi F, Piergili Fioretti D (2006). Prevalence and genotyping of Giardia duodenalis from FIV + cats in Central Italy. Parassitologia 48:320

Doglioni C, De Boni M, Cielo R, Laurino L, Pelosio P, Braidotti P, Viale G (1992) Gastric giardiasis. J Clin Pathol 45:964-967

Faustini A, Marinacci C, Fabrizi E, Marangi M, Recchia O, Pica R, Giustini F, La Marca A, Nacci A, Panichi G, Perucci CA (2006) The impact of the Catholic Jubilee in 2000 on infectious diseases. A case-control study of giardiasis, Rome, Italy 2000-2001. Epidemiol Infect 134:649-668

Fayer R (2004). Cryptosporidium: a water-borne zoonotic parasite. Vet Parasitol 126:37-56

Fayer R, Dubey JP, Lindsay DS (2004) Zoonotic protozoa: from land to sea. Trends Parasitol 20:531-536

Frost FJ, Fea E, Gilli G, Biorci F, Muller TM, Craun GF, Calderon RL (2000). Serological evidence of Cryptosporidium infections in Southern Europe. Eur J Epidemiol 16:385-390

Frost FJ, Muller TB, Calderon RL, Craun GF (2004) Analysis of serological responses to Cryptosporidium antigen among NHANES III participants. Ann Epidemiol 14:473-478

Frost FJ, Roberts M, Kunde TR, Craun G, Tollestrup K, Harter L, Muller T (2005a) How clean must our drinking water be: the importance of protective immunity. J Infect Dis 191:809-814

Frost FJ, Tollestrup K, Craun GF, Fairley CK, Sinclair MI, Kunde TR (2005b) Protective immunity associated with a strong serological response to a Cryptosporidium-specific antigen group, in HIVinfected individuals. J Infect Dis 192:618-621

Galiero G, Consalvo F, Carullo M (1994) La criptosporidiosi nei vitelli bufalini: un aggiornamento. Sel Vet 35:445-449

Gatti S, Lopes R, Cevini C, Ijaoba B, Bruno A, Bernuzzi AM, de Lio P, Monco A, Scaglia M (2000) Intestinal parasitic infections in an institution for the mentally retarded. Ann Trop Med Parasitol 94:453-460

Genchi G, Hermon I, Sangalli G, Traldi G (1984) La criptosporidosi, fattore determinante nella diarrea neonatale. Praxis Vet 5:5-8

Giacometti A, Cirioni O, Balducci M, Drenaggi D, Quarta M, De Federicis M, Ruggeri P, Colapinto D, Ripani G, Scalise G (1997) 
Epidemiologic features of intestinal parasitic infections in Italian mental institutions. Eur J Epidemiol 13:825-830

Giacometti A, Cirioni O, Fortuna M, Drenaggi D, Veccia S, D’Errico MM, Scalise G (2000) Giardiasis: a parasitic disease of continued topicality. Study of prevalence among a selected adult population. Infez Med 8:82-86

Giangaspero A, Traldi G, Paoletti B, Traversa D, Bianciardi P (2002) Efficacy of pyrantel embonate, febantel and praziquantel against Giardia spp. in naturally infected adult dogs. Vet Rec 150:184-186

Giangaspero A, Molini U, Iorio R, Traversa D, Paoletti B, Giansante C (2005a) Cryptosporidium parvum oocysts in seawater clams (Chamelea gallina) in Italy. Prev Vet Med 69:203-212

Giangaspero A, Paoletti B, Iorio R, Traversa D (2005b) Prevalence and molecular characterization of Giardia duodenalis from sheep in central Italy. Parasitol Res 96:32-37

Giangaspero A, Iorio R, Paoletti B, Traversa D, Capelli G (2006) Molecular evidence for Cryptosporidium infection in dogs in Central Italy. Parasitol Res 99:297-299

Gomez Morales MA, Pozio E, Croppo GP (1992) Serodiagnosis of cryptosporidiosis in Italian HIV-positive patients by means of an oocyst soluble antigen in an ELISA. J Infect 25:229-236

Gomez-Couso H, Freire-Santos F, Martinez-Urtaza J, Garcia-Martin O, Ares-Mazas ME (2003) Contamination of bivalve molluscs by Cryptosporidium oocysts: the need for new quality control standards. Int J Food Microbiol 87:97-105

Gomez-Couso H, Mendez-Hermida F, Castro-Hermida JA, AresMazas E (2006) Cooking mussels (Mytilus galloprovincialis) by steam does not destroy the infectivity of Cryptosporidium parvum. J Food Prot 69:948-950

Grana L, Lalle M, Habluetzel A, Silvestri S, Traldi G, Tonanti D, Pozio E, Cacciò SM (2006) Distibution of zoonotic and animal specicif genotypes of Cryptosporidium and Giardia in calves of cattle farms in the Marche region. Parassitologia 48:208

Grazioli B, Matera G, Laratta C, Schipani G, Guarnieri G, Spiniello E, Imeneo M, Amorosi A, Foca A, Luzza F (2006) Giardia lamblia infection in patients with irritable bowel syndrome and dyspepsia: a prospective study. World J Gastroenterol 12:1941-1944

Hill SL, Cheney JM, Taton-Allen GF, Reif JS, Bruns C, Lappin MR (2000) Prevalence of enteric zoonotic organisms in cats. J Am Vet Med Ass 216:687-692

Horman A, Korpela H, Sutinen J, Wedel H, Hanninen ML (2004) Meta-analysis in assessment of the prevalence and annual incidence of Giardia spp. and Cryptosporidium spp. infections in humans in the Nordic countries. Int J Parasitol 34:1337-1346

Hunter PR, Thompson RC (2005) The zoonotic transmission of Giardia and Cryptosporidium. Int J Parasitol 35:1181-1190

ISTAT (2005) Available at: http://www.istat.it/dati/catalogo/20060524 00/ rapporto2005.pdf

Joachim A (2004) Human cryptosporidiosis: an update with special emphasis on the situation in Europe. J Vet Med B Infect Dis Vet Public Health 51:251-259

Lalle M, Pozio E, Capelli G, Bruschi F, Crotti D, Cacciò SM (2005) Genetic heterogeneity at the beta-giardin locus among human and animal isolates of Giardia duodenalis and identification of potentially zoonotic subgenotypes. Int J Parasitol 35:207-213

Lalle M, Frangipane di Regalbono A, Poppi L, Nobili G, Tonanti M, Pozio E, Simone M, Cacciò S (2007) A novel Giardia duodenalis A subtype in fallow deer from Northern Italy. J Parasitol 93:426-428

Libanore M, Bicocchi R, Rossi MR, Montanari P, Sighinolfi L, Macario F, Ghinelli F (1991) Incidence of giardiasis in adults patients with acute enteritis. Minerva Med 82:375-380

Lonigro A, Pollice A, Spinelli R, Berrilli F, Di Cave D, D’Orazi C, Cavallo P, Brandonisio O (2006) Giardia cysts and Cryptospo- ridium oocysts in membrane-filtered municipal wastewater used for irrigation. Appl Environ Microbiol 72:7916-7918

Maggi P, Larocca AM, Quarto M, Serio G, Brandonisio O, Angarano G, Pastore G (2000) Effect of antiretroviral therapy on cryptosporidiosis and microsporidiosis in patients infected with human immunodeficiency virus type 1. Eur J Clin Microbiol Infect Dis 19:213-217

Maggi P, Larocca AM, Ladisa N, Carbonara S, Brandonisio O, Angarano G, Pastore G (2001) Opportunistic parasitic infections of the intestinal tract in the era of highly active antiretroviral therapy: is the $\mathrm{CD} 4(+)$ count so important? Clin Infect Dis 33:1609-1611

Magi B, Canocchi V, Tordini G, Cellesi C, Barberi A (2006) Cryptosporidium infection: diagnostic techniques. Parasitol Res 98:150-152

Mandelli A, Valeri A (1972). Coccidi del genere Cryptosporidium in alcune specie aviarie. Atti XI Conv. Pat Aviare 2:239-245

Manfredi R (2000) Effects of the introduction of highly active antiretroviral therapy (HAART) on the temporal trend of diagnosis of AIDS and AIDS-defining disorders. Infez Med 8:134-138

Manfredi MT, Preti R, Lalle M, Tonanzi D, Pozio E, Cacciò SM (2006) Cryptosporidium spp. infections in pigs from industrial farms of northern Italy: prevalence and genotyping. Parassitologia 48:269

Mastandrea G, Micarelli A (1968) Ricerca parassitaria nei prodotti vegetali prelevati da alcuni mercati rionali della città di Roma. Arch Ital Sci Med Trop Parassitol 49:55-59

Mattei N, Pellizzato M (1997) Mollusk fisheries and aquaculture in Italy. In: The history, present condition, and future of the molluscan fisheries of North and Central America and Europe. Volume 3, Europe. U.S. Dep. Commer., NOAA Tech. Rep. Fishery Bulletin 129:201-216

McGlade TR, Robertson ID, Elliot AD, Thompson RC (2003) High prevalence of Giardia detected in cats by PCR. Vet Parasitol 110:197-205

Mele R, Gomez Morales MA, Tosini F, Pozio E (2003) Indinavir reduces Cryptosporidium parvum infection in both in vitro and in vivo models. Int J Parasitol 33:757-764

Molini U, Iorio R, Traversa D, Paoletti B, Giansante C, Giangaspero A (2004) Rilievo di Giardia spp. e Cryptosporidium spp. nelle vongole (Chamelea gallina) della costa abruzzese. Ittiopatologia $1: 34-40$

Molini U, Traversa D, Ceschia G, Iorio R, Boffo L, Zentilin A, Capelli G, Giangaspero A (2007) Temporal occurrence of Cryptosporidium in the Asian clam Ruditapes philippinarum in the Northern Adriatic Italian Lagoons. J Food Prot 70:494-499

Moretti MV, Fiorio M, Antonelli S, Baldelli F, Stagni G, Pauluzzi S (1988) Study on the presence of Cryptosporidium in Umbria. Boll Ist Sieroter Milan 67:226-231

Oliveri R, Di Piazza F, Marsala B, Cerame G, Firenze A, Di Benedetto MA (2006) Occurrence of Giardia cysts and Cryptosporidium oocysts in swimming pools in the province of Palermo, Italy. Ann Ig 18:367-374

Paoletti B, Iorio R, Traversa D, Gatti A, Capelli G, Giangaspero A (2006) Trends in the epidemiology of giardiosis in dogs from Abruzzo region. Parassitologia 48:270

Papini R, Gorini G, Spaziani A, Cardini G (2005) Survey on giardiosis in shelter dog populations. Vet Parasitol 128:333-339

Papini R, Cardini G, Paoletti B, Giangaspero A (2007a) Detection of Giardia Assemblage A in cats in Florence, Italy. Parasitol Res 100:653-656

Papini R, Giuliani G, Gorini G, Cardini G (2007b). Survey of Feline Giardiasis by ELISA test in Italy. Vet Res Comm 31:297-303 
Piergili-Fioretti D, Moretti A (1989) Giardiasis in dogs: preliminary studies on its frequency in Umbria. Biol Oggi 3:7-9

Piergili-Fioretti D, Moretti A, Sacconi G (1991) Intestinal cryptosporidiosis of roaster and boiler chicken flocks in central Italy. Acta Med Vet 37:149-157

Pozio E, Rezza G, Boschini A, Pezzotti P, Tamburrini A, Rossi P, Di Fine M, Smacchia C, Schiesari A, Gattei E, Zucconi R, Ballarini $P$ (1997) Clinical cryptosporidiosis and human immunodeficiency virus (HIV)-induced immunosuppression: findings from a longitudinal study of HIV-positive and HIV-negative former injection drug users. J Infect Dis 176:969-975

Pucci R, Marino R, Ilardi I (1967) Giardiasis in childhood. Arch Ital Sci Med Trop Parassitol 48:135-144

Rinaldi L, Musella V, Condoleo R, Saralli G, Veneziano V, Bruni G, Condoleo RU, Cringoli G (2006) Giardia and Cryptosporidium in water buffaloes (Bubalus bubalis) Parasitol Res 100:11131118

Rosso L, Archilei R, Cenci Goga B (1989) Indagine sulla presenza di cisti di Giardia in feci di cani clinicamente sani mediante immunofluorescenza. Atti Soc It Sci Vet 43:1271-1274

Sacco C, Bianchi M, Lorini C, Burrini D, Berchielli S, Lanciotti E (2006) Removal of Cryptosporidium and Giardia in drinking water treatment in a Tuscan area. Ann Ig 18:117-126

Saralli G, Condoleo RU, Centra A, Scissione U, Bernardi C (2001) Diffusione di Cryptosporidium parvum in bufali allevati in provincia di Latina. Atti I Congresso Nazionale sull'Allevamento del Bufalo 1:420-423

Savioli L, Smith H, Thompson A (2006) Giardia and Cryptosporidium join the 'Neglected Diseases Initiative'. Trends Parasitol 22:203-208

Scotti S, Pettoello Mantovani M, Polito G, Carlomagno F, Coppola A, di Martino L (1996). Giardia duodenalis infections in pediatrics: our series. Infez Med 4:35-40

Smith HV, Caccio SM, Tait A, McLauchlin J, Thompson RC (2006) Tools for investigating the environmental transmission of
Cryptosporidium and Giardia infections in humans. Trends Parasitol 22:160-167

Sorgi C, Cavallaio M, Brianti E, Ferlazzo M, Giannetto S (2007) Cryptosporidium and Giardia in mussels (Mytilus galloprovincialis) from Faro salt-lake, Sicily. Parassitologia 48:296

Spinelli R, Brandonisio O, Serio G, Trerotoli P, Ghezzani F, Carito V, Dajci N, Doci A, Picaku F, Dentico P (2006) Intestinal parasites in healthy subjects in Albania. Eur J Epidemiol 21:161-166

Stark D, van Hal S, Marriott D, Ellis J, Harkness J (2007) Irritable bowel syndrome: A review on the role of intestinal protozoa and the importance of their detection and diagnosis. Int J Parasitol 37:11-20

Thompson RC, Monis PT (2004) Variation in Giardia: implications for taxonomy and epidemiology. Adv Parasitol 58:69-137

Traldi G (1990) Report of Cryptosporidium in an adult dog. Atti S.I.S. Vet 29:1293-1294

Traldi G, Castiglioni G (1993) Giardia negli animali da affezione. Una parassitosi da non sottovalutare. Obiettivi Doc Vet 10:69-70

Traub RJ, Monis PT, Robertson I, Irwin P, Mencke N, Thompson RC (2004) Epidemiological and molecular evidence supports the zoonotic transmission of Giardia among humans and dogs living in the same community. Parasitology 128:253-262

Traversa D, Giangaspero A, Molini U, Iorio R, Paoletti B, Otranto D, Giansante C (2004). Genotyping of Cryptosporidium isolates from Chamelea gallina clams in Italy. Appl Environ Microbiol 70:4367-4370

Vasilopulos RJ, Mackin AJ, Rickard LG, Pharr GT, Huston CL (2006) Prevalence and factors associated with fecal shedding of Giardia spp. in domestic cats. J Am Anim Hosp Assoc 42:424-429

Veronesi F, Diaferia M, Piergili Fioretti D (2006) Epidemiology of Cryptosporidium and Giardia infections in foals of central Italy. Parassitologia 48:275

Xiao L, Ryan UM (2004) Cryptosporidiosis: an update in molecular epidemiology. Curr Opin Infect Dis 17:483-490

Yu JR, Choi SD (2000) The effect of microfilament inhibitor on the Cryptosporidium infection in vitro. Korean J Parasitol 38:257-261 